BMJ Open

Diabetes

Research

\& Care

\title{
SGLT2 inhibitors in patients with type 2 diabetes with non-alcoholic fatty liver diseases: an umbrella review of systematic reviews
}

\author{
Shih-Chieh Shao, ${ }^{1,2}$ Liang-Tseng Kuo, ${ }^{3,4}$ Rong-Nan Chien, , ${ }^{4,6}$ Ming-Jui Hung, 4,7 \\ Edward Chia-Cheng Lai (1) ${ }^{2,8}$
}

To cite: Shao S-C, Kuo L-T, Chien R-N, et al. SGLT2 inhibitors in patients with type 2 diabetes with non-alcoholic fatty liver diseases: an umbrella review of systematic reviews. BMJ Open Diab Res Care 2020;8:e001956. doi:10.1136/ bmjdrc-2020-001956

- Supplemental material is published online only. To view please visit the journal online (http://dx.doi.org/10.1136/ bmjdrc-2020-001956).

Received 20 0ctober 2020 Revised 3 November 2020 Accepted 6 November 2020
Check for updates

(c) Author(s) (or their employer(s)) 2020. Re-use permitted under CC BY-NC. No commercial re-use. See rights and permissions. Published by BMJ.

For numbered affiliations see end of article.

Correspondence to Dr Edward Chia-Cheng Lai; edward_lai@mail.ncku.edu.tw

\section{ABSTRACT}

Introduction Sodium glucose co-transporter 2 (SGLT2) inhibitors have been reported to benefit liver functions in patients with type 2 diabetes (T2D) with non-alcoholic fatty liver disease (NAFLD). The aim of this study is to critically appraise existing systematic reviews in order to consolidate evidence associating the use of SGLT2 inhibitors with beneficial hepatic results for patients with T2D with NAFLD.

Methods This umbrella review searched relevant published systematic reviews of clinical trials from PubMed and Embase between inception and September 16, 2020. Two independent investigators appraised study quality using AMSTAR2 (Assessment of Multiple Systematic Reviews 2). The hepatic effects from SGLT2 inhibitors were summarized based on liver enzymes, liver fat, liver histology, liver cirrhosis and liver cancer.

Results 0 25 screened potential systematic reviews, we ultimately included 7 in this study. However, none of them could be rated as being of high methodological quality. Five systematic reviews indicated that SGLT2 inhibitors could effectively decrease liver fat and liver parameters of alanine aminotransferase and gamma-glutamyl transferase in patients with NAFLD. Two systematic reviews indicated that SGLT2 inhibitors could reduce hepatosteatosis, as supported by biopsy-proven evidence of improvement from a small clinical trial, but no evidence of liver fibrosis improvement was found.

Conclusions There is some association between SGLT2 inhibitor use and observed benefits to liver functions in patients with T2D with NAFLD, although the quality of current systematic reviews remains relatively low. Further evaluation of long-term liver outcomes with SGLT2 inhibitors in cases of liver cirrhosis and liver cancer is warranted.

\section{BACKGROUND}

Type 2 diabetes (T2D) and non-alcoholic fatty liver disease (NAFLD) are both common and frequently occur together, whereby they can act synergistically, furthering undesirable outcomes. ${ }^{1}$ The prevalence of NAFLD among patients with T2D was $55.5 \%,{ }^{2}$ and one out of five patients with T2D with normal liver functions had biopsy-proven non-alcoholic

\section{Significance of this study}

What is already known about this subject?

- Type 2 diabetes (T2D) is closely related to nonalcoholic liver disease (NAFLD) and probably increases the risk of cirrhosis and hepatocellular carcinoma.

- Several systematic reviews have reported sodium glucose co-transporter 2 (SGLT2) inhibitor, a novel antihyperglycemic medication, has beneficial liver effects in patients with T2D with NAFLD; however, comprehensive review of the current evidence available on SGLT2 inhibitors and liver outcomes remains unclear.

What are the new findings?

- SGLT2 inhibitors could improve liver enzymes, including alanine aminotransferase, gamma-glutamyl transferase, and liver fat, in patients with T2D with NAFLD.

- SGLT2 inhibitors could reduce hepatosteatosis, as supported by biopsy-proven evidence of improvement from a small clinical trial, but no evidence of liver fibrosis improvement was found.

\section{How might these results change the focus of} research or clinical practice?

- The use of SGLT2 inhibitors is a potentially rational option for patients with T2D with NAFLD since current evidence supports that SGLT2 inhibitor use is associated with liver enzyme and liver fat reduction in patients with T2D with NAFLD.

- Further studies conforming to high scientific standards are required to bridge the gap in evidence regarding SGLT2 inhibitor use and long-term liver outcomes, such as liver cirrhosis and liver cancer.

steatohepatitis (NASH). ${ }^{3}$ The risk of developing cardiovascular complications of diabetes is increased when both T2D and NAFLD are present, as is the risk of greater severity of NAFLD, such as liver cirrhosis, liver cancer and death. ${ }^{4-6}$ 
Pharmacotherapies for patients with T2D with NAFLD have not been established. Metformin, the first-line antidiabetes medication in T2D, is generally not suggested as specific therapy for NAFLD because its beneficial effect on liver histology has not been proven. ${ }^{7}$ Although pioglitazone has been shown to have favorable effects on NASH histology, ${ }^{8}$ side effects including weight gain, retention of fluid, cancer occurrence and bone fracture limit its clinical use. ${ }^{9}$ Some studies have indicated that dipeptidyl peptidase 4 inhibitors and glucagon-like peptide 1 receptor agonists were of benefit to patients' liver functions. However, only a few of these studies supported their use in patients with T2D with NAFLD.$^{10-12}$ Therefore, lifestyle modifications, including diet and physical activity, remain the mainstay of current therapy for patients with T2D with NAFLD. ${ }^{13} 14$

Sodium glucose co-transporter 2 (SGLT2) inhibitors are novel antidiabetic medications that increase the excretion of urinary glucose and thus lower blood glucose levels. In addition to their hypoglycemic effects, SGLT2 inhibitors have been shown to offer several favorable pleiotropic effects on patients' body weight and liver enzymes that may potentially improve or prevent the progression of NAFLD. ${ }^{15} 16$ Although some systematic reviews and meta-analyses have demonstrated the hepatic benefits from SGLT2 inhibitors in patients with T2D with NAFLD, the quality of these systematic reviews has not been evaluated. ${ }^{17}$

Umbrella review is a useful tool to gain comprehensive overview of systematic reviews and meta-analyses published on a specific topic by examining the studies' strengths and risks of bias. ${ }^{18}$ We performed an umbrella review to critically appraise existing systematic reviews to consolidate evidence of associations between use of SGLT2 inhibitors and beneficial hepatic effects in patients with T2D with NAFLD and to identify current unmet needs with regard to this topic.

\section{METHODS}

\section{Search strategy}

We searched PubMed and Embase to identify systematic reviews of SGLT2 inhibitors for patients with T2D with NAFLD published between inception and September 16, 2020. Searches were supplemented by manually reviewing reference lists to find relevant articles in included systematic reviews. The search strategy combined selected keywords (eg, SGLT2 inhibitors and NAFLD) with MeSH or Emtree terms and directed clinical queries for systematic reviews (eg, systematic (sb) in PubMed). Languages were not restricted. We present the full search strategy applied to the two databases in online supplemental table 1.

\section{Screening and final selection of systematic reviews}

The articles to be included were independently selected by two reviewers (S-CS and L-TK) following the criteria defined in the Preferred Reporting Items for Systematic
Reviews and Meta-Analyses (PRISMA) statement (online supplemental table 2). Articles eligible for inclusion were systematic reviews as identified by presentation consistent with the PRISMA statement, ${ }^{19}$ examining the effectiveness of SGLT2 inhibitors for T2D with NAFLD in clinical trials. We did not include references from animal studies or conference abstracts. Full-text articles of all references that appeared relevant were assessed individually, whereby disagreements between the reviewers were resolved by discussion.

\section{Data extraction}

The same reviewers then extracted relevant data from the systematic reviews selected. The main information thus retrieved from each review was then classified as relating to study authors and year, number of included studies and participants, country, exposure, duration and main findings. It is presented in a table.

\section{Assessment of methodological quality}

Assessment of the methodological quality of the systematic reviews selected for inclusion was performed by two independent reviewers (S-CS and L-TK), following the 'Assessment of Multiple Systematic Reviews 2' (AMSTAR2) criteria. These criteria offer a broadly used and reproducible way to evaluate systematic reviews of randomized controlled trials for their methodological quality. ${ }^{20}$ The AMSTAR2 assessment is derived from the results of 16 inquiries (domains) covering issues such as the following: evidence of a review protocol having been registered prior to study commencement; whether literature search is conducted to an adequate degree; where individual studies are excluded, the provision of justification therefor; acknowledgment and assessment of possible bias arising in the studies; the suitability of applied meta-analytic procedures; to what degree bias risks are taken into consideration in the interpretation of review results; and whether publication bias might arise and its likely impact. The responses are 'Yes' for positive or 'No' for negative or unknown, with 'Partial Yes' indicating incomplete adherence to the criteria. After discussion, the two reviewers (S-CS and L-TK) awarded each study a rating for its methodological quality. The ratings were 'high', 'moderate', 'low' or 'critically low', based on the outcome of the AMSTAR2 inquiries.

\section{Data analysis}

We carried out a descriptive analysis for the included systematic reviews. We summarized the conclusions drawn by the systematic reviews regarding SGLT2 inhibitors and the liver outcomes of interest, including liver enzymes (eg, aspartate aminotransferase, AST; alanine aminotransferase, ALT; gamma-glutamyl transferase, GGT), liver fat, histology, cirrhosis and liver cancer. Whenever more than one systematic review had been performed on the same liver outcome, we examined whether the main reported conclusions were concordant. 


\section{RESULTS}

We found a total of 25 records by applying the keywords described aforementioned in PubMed and Embase. After removing duplicates and examining titles and abstracts, we found seven systematic reviews of clinical trials that met the study inclusion criteria. We present the selection process by PRISMA chart in figure 1 .

\section{Quality assessment}

The application of the AMSTAR2 assessment standard yielded 'moderate' confidence in the review results for only one of the systematic reviews included, meaning it had more than one non-critical weakness. 'Low' confidence was found for the results of three of the reviews, implying they had one critical flaw, regardless of other non-critical weaknesses. 'Critically Low' was the AMSTAR2 rating for confidence in the results of the remaining three reviews, meaning they displayed more than one critical flaw, regardless of non-critical weaknesses (table 1). Analysis of the 16 domain responses revealed that of the seven included systematic reviews, six were negative with regard to the same four domains: provision of a protocol prior to review commencement (second domain); listing excluded studies and justifying their exclusion (seventh domain); reporting any funding sources of the studies they included (tenth domain); and evaluating how the risk of bias in individual studies might impact the interpretation of the results (thirteenth domain). By contrast, all the systematic reviews included were positive for the following three domains: presentation of research questions and inclusion criteria (first domain); selection of study design to be included (third domain); and potential conflicts of interest and funding sources (sixteenth domain).

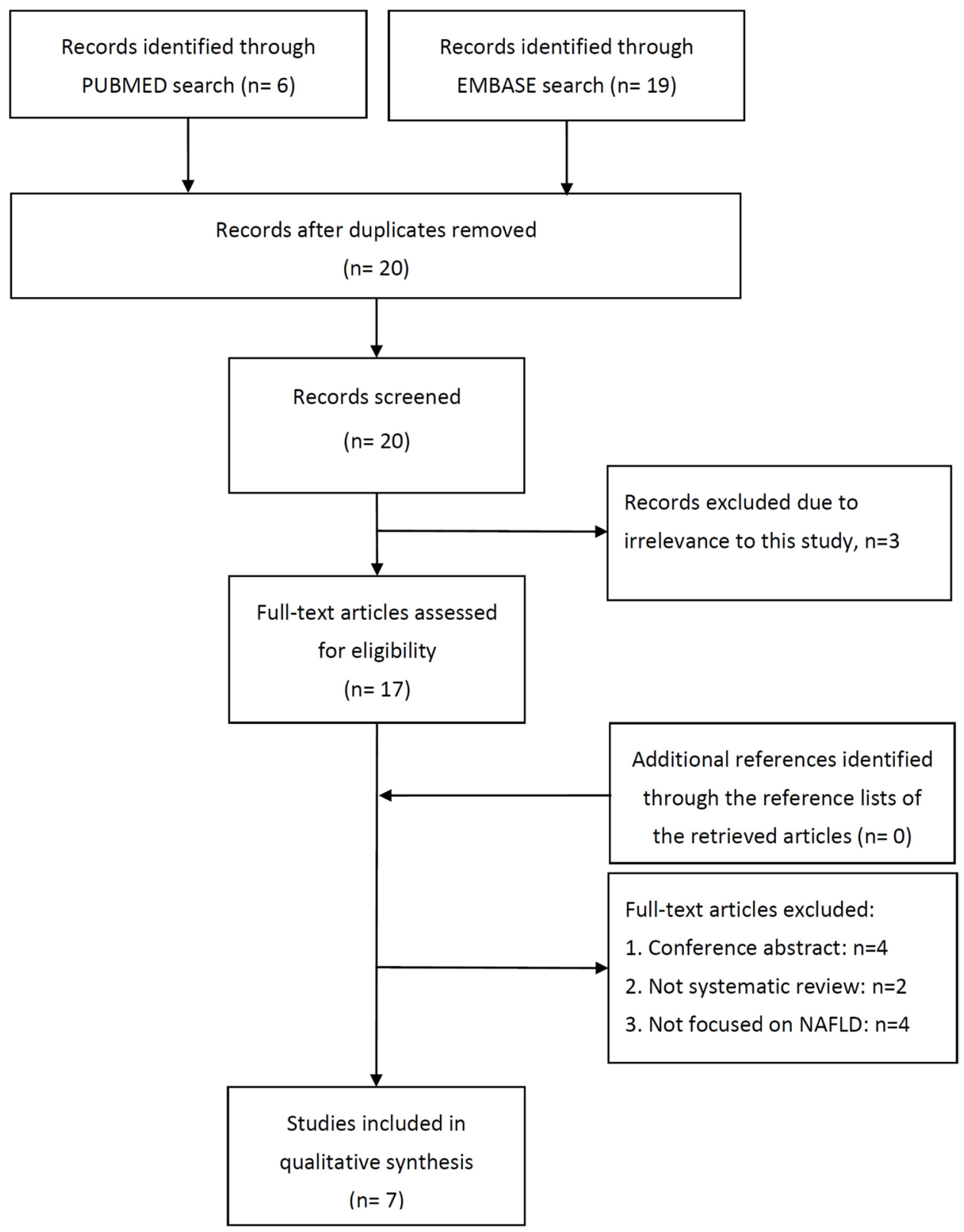

Figure 1 Literature screening and selection process. NAFLD, non-alcoholic fatty liver disease. 
Table 1 Methodological quality of systematic reviews based on AMSTAR2 criteria

\begin{tabular}{|c|c|c|c|c|c|c|c|c|c|c|c|c|c|c|c|c|c|}
\hline First author (year) & Q1 & Q2 & Q3 & Q4 & Q5 & Q6 & Q7 & Q8 & Q9 & Q10 & Q11 & Q12 & Q13 & Q14 & Q15 & Q16 & Overall quality \\
\hline Kumar $(2020)^{21}$ & $Y$ & $\mathrm{~N}$ & $\mathrm{Y}$ & PY & $Y$ & $\mathrm{~N}$ & $\mathrm{~N}$ & PY & $\mathrm{N}$ & $\mathrm{N}$ & $\mathrm{Y}$ & $\mathrm{N}$ & $\mathrm{N}$ & $\mathrm{Y}$ & $\mathrm{N}$ & $Y$ & Critically low quality \\
\hline Dougherty $(2020)^{25}$ & $\mathrm{Y}$ & $\mathrm{N}$ & $\mathrm{Y}$ & PY & $\mathrm{Y}$ & $\mathrm{N}$ & $\mathrm{N}$ & $\mathrm{Y}$ & $\mathrm{N}$ & $\mathrm{N}$ & $x$ & $x$ & $\mathrm{~N}$ & $\mathrm{~N}$ & $x$ & $\mathrm{Y}$ & Critically low quality \\
\hline Xing $(2020)^{23}$ & Y & $\mathrm{N}$ & $\mathrm{Y}$ & PY & $Y$ & Y & $\mathrm{N}$ & PY & Y & $\mathrm{N}$ & Y & $\mathrm{N}$ & $\mathrm{N}$ & Y & Y & Y & Low quality \\
\hline Mantovani $(2020)^{39}$ & $\mathrm{Y}$ & $\mathrm{N}$ & $\mathrm{Y}$ & PY & $\mathrm{Y}$ & $\mathrm{Y}$ & $\mathrm{Y}$ & PY & PY & $\mathrm{N}$ & $x$ & $x$ & $\mathrm{Y}$ & $\mathrm{Y}$ & $x$ & $\mathrm{Y}$ & Moderate quality \\
\hline $\begin{array}{l}\text { Pan and Stanley } \\
(2020)^{24}\end{array}$ & $Y$ & $\mathrm{~N}$ & $Y$ & $\mathrm{~N}$ & $\mathrm{~N}$ & $\mathrm{~N}$ & $\mathrm{~N}$ & PY & $\mathrm{N}$ & $\mathrm{N}$ & $x$ & $x$ & $\mathrm{~N}$ & $\mathrm{~N}$ & $x$ & $Y$ & Critically low quality \\
\hline $\operatorname{Raj}(2019)^{40}$ & $\mathrm{Y}$ & Y & Y & PY & $\mathrm{Y}$ & $\mathrm{N}$ & $\mathrm{N}$ & PY & Y & $\mathrm{Y}$ & $x$ & $x$ & $\mathrm{~N}$ & $\mathrm{~N}$ & $x$ & $\mathrm{Y}$ & Low quality \\
\hline Tang $(2016)^{22}$ & $Y$ & $\mathrm{~N}$ & $Y$ & PY & $\mathrm{N}$ & $Y$ & $\mathrm{~N}$ & PY & PY & $\mathrm{N}$ & $X$ & $x$ & $\mathrm{~N}$ & $Y$ & $Y$ & $Y$ & Low quality \\
\hline
\end{tabular}

Q1: Were patient/problem, intervention, comparsion and outcome (PICO) components taken into consideration when drawing up the research questions and inclusion criteria? Q2: Does the review report explicitly state that the methods of the review were decided before commencing the review, and if these methods were changed does the report justify the change? Q3: Have the authors provided an explanation for their choice of the study designs they include in the review? Q4: Was the authors' strategy for literature search sufficiently comprehensive? Q5: Were any duplicates of studies selected? Q6: Were any duplicate data extracted? Q7: Were excluded studies listed and a justification given for the exclusion? Q8: Is the detail with which the authors describe the studies adequate to the task? Q9: Has the risk of bias in individual studies been addressed satisfactorily through an appropriate technique? Q10: Are the individual studies' funding sources reported in the review? Q11: Have the authors of the review employed appropriate methods to statistically combine the results of any meta-analysis conducted? Q12: Have the review authors assessed what impact risk of bias in individual studies might have on the results of their meta-analyses or on other evidence formation? Q13: Is risk of bias in the individual studies adequately addressed by the authors in the discussion and interpretation of their review's results? Q14: Where heterogeneity was observed in the review results, have the authors attempted a satisfactory discussion and explanation thereof? Q15: If quantitative synthesis was performed, have the authors adequately addressed the issue of publication bias (small study bias) and offered a discussion of how it might be likely to impact the review results? Q16: Have the review authors reported on any conflicts of interest, including through funding of their review, which might arise?

AMSTAR2, Assessment of Multiple Systematic Reviews 2; N, no: negative response or response not available; PY, partial yes: incomplete adherence to the criteria; $\mathrm{X}$, no meta-analyses performed; $\mathrm{Y}$, yes: positive response.

\section{Changes in liver enzymes}

In four out of seven systematic reviews reporting SGLT2 inhibitors' effects on liver enzymes, all, three and two studies reported the changes of ALT, AST and GGT levels, respectively (table 2). Qualitative findings of included systematic reviews indicated that SGLT2 inhibitors could effectively reduce these liver parameters in patients with NAFLD, compared with the placebo and other active comparators. However, the meta-analysis by Kumar et $a l^{21}$ showed that, to achieve significance, SGLT2 inhibitors could decrease ALT and GGT levels by $-16.17 \mathrm{U} / \mathrm{L}$ (95\% CI -21.74 to -10.60$)$ and $-19.31(95 \%$ CI -21.13 to -17.49 ) while yielding no statistical difference in AST reduction ( $-7.09 \mathrm{U} / \mathrm{L}, 95 \% \mathrm{CI}-17.03$ to 2.85$)$. Notably, moderate to high heterogeneity among the clinical trials of SGLT2 inhibitors and ALT/AST evaluations was found.

\section{Changes in liver fat}

Four systematic reviews reported changes in liver fat from SGLT2 inhibitors (table 2). The first systematic review from Tang $e t a l^{22}$ reported no difference in hepatic fat content improvement between dapagliflozin and placebo (absolute change from baseline assessed by proton magnetic resonance spectroscopy, $-2.4 \%$ vs $-1.5 \%$ ), based on findings from a single randomized controlled trial. However, the more recent meta-analysis from Xing et $a l^{23}$ including two randomized controlled trials, found SGLT2 inhibitors could further reduce liver fat content by $-2.07 \%$ (95\% CI -3.86 to -0.28$)$ calculated by MRI proton density fat fraction, compared with metformin or placebo. Notably, another systematic review from
Pan and Stanley ${ }^{24}$ showed SGLT2 inhibitors moderately improved liver fat content, as determined by the liver to spleen attenuation ratio, but the effects were similar to the pioglitazone comparator (absolute change from baseline: $-0.22 \pm 0.04$ vs $-0.21 \pm 0.03$ ) in a randomized, open-label, controlled clinical trial.

\section{Changes in liver histology, liver cancer and liver cirrhosis}

We found a systematic review by Dougherty et $a l^{25}$ reported SGLT2 inhibitors improved liver histology in patients with NASH according to findings from a single-arm clinical trial (table 2). However, the recent systematic review by Kumar $e t a l^{21}$ demonstrated SGLT2 inhibitors may not reduce liver fibrosis (standard mean difference: -0.07 , $95 \%$ CI -0.33 to 0.19$)$. No systematic review summarized the effects on liver cirrhosis and liver cancer after SGLT2 inhibitor treatment.

\section{DISCUSSION}

This umbrella review summarizes existing systematic reviews for the purpose of consolidating dispersed evidence of associations between use of SGLT2 inhibitors and liver outcomes in patients with T2D with NAFLD. We found the systematic reviews demonstrated that the use of SGLT2 inhibitors was associated with reductions in liver enzymes and liver fat in patients with T2D with NAFLD. Notably, a clinical trial from one of the systematic reviews has proven histological improvement of NASH from liver biopsies among patients receiving SGLT2 inhibitors. ${ }^{26}$ However, no sufficient evidence of SGLT2 inhibitors was 


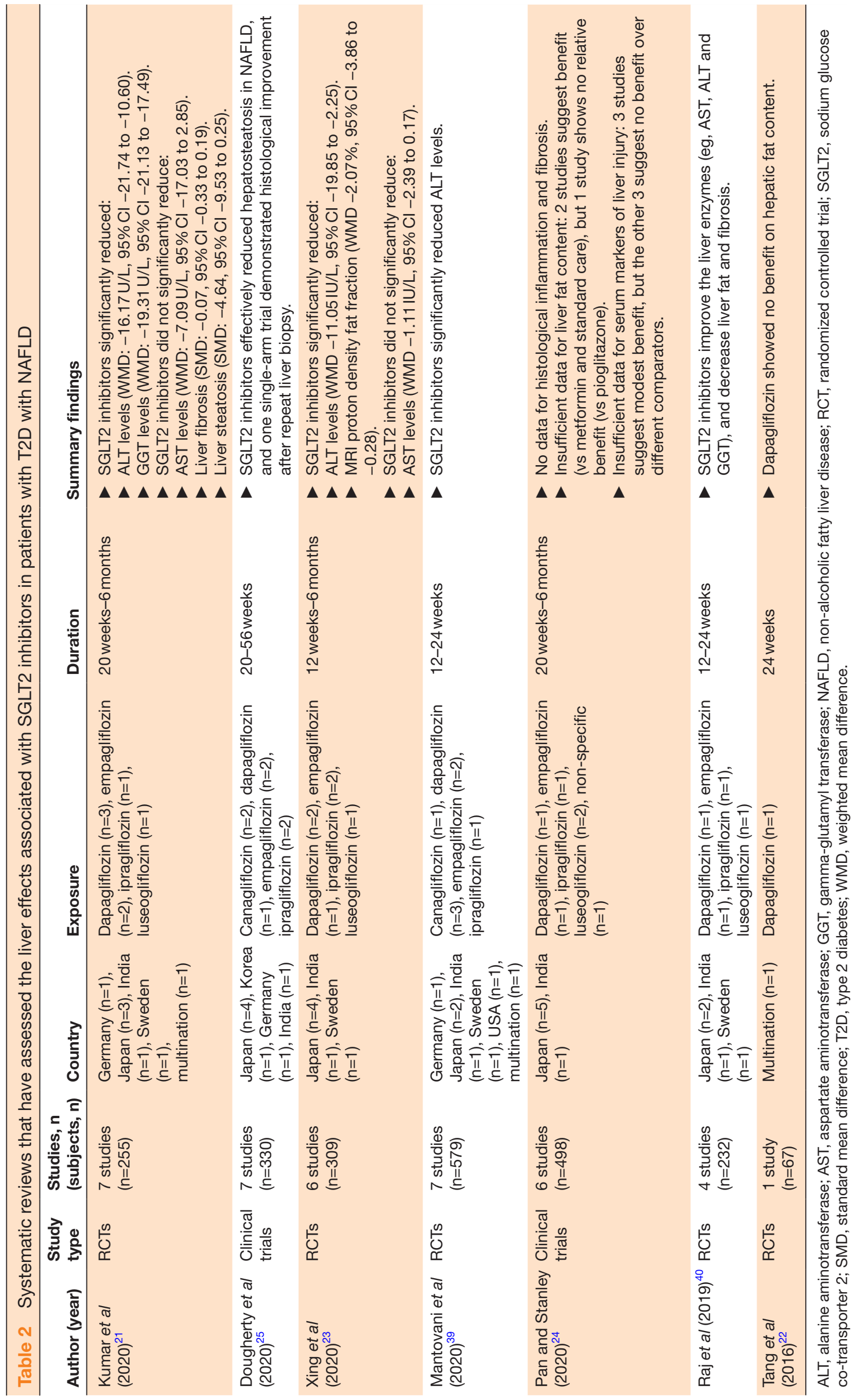


seen to significantly improve liver fibrosis. We found no study analyzing the association between SGLT2 inhibitor use and incidence of liver cirrhosis and cancer. On the basis of evaluation by AMSTAR2, we found current systematic reviews to be of moderate and critically low quality, suggesting the need for further investigation. Although existing systematic reviews support that SGLT2 inhibitor use may offer beneficial effects on hepatic functions in patients with T2D with NAFLD, three major issues are noteworthy.

First, the findings from the systematic reviews were based on clinical trials and may not be applicable to patients in a real-world setting, ${ }^{27} 28$ because patients with hepatic impairment (eg, ALT >2.5-3 times the upper reference limit) were not included in the clinical trials. Additional real-world evidence is required to address this limitation. For example, a recent retrospective multiinstitutional cohort study, which included patients with hepatic impairment, demonstrated that the beneficial effects on hepatic functions remained consistent. ${ }^{16}$ In addition, given the liver benefits from SGLT2 inhibitors in patients with NAFLD have been proven by clinical trials with the relatively short observational period (most within 56 weeks), future studies with longer follow-up of patients are warranted.

Second, the underlying mechanisms which drive liver function improvement through SGLT2 inhibitors in patients with T2D with NAFLD remain unclear. Some studies have suggested the decrease in liver enzymes and liver fat is related to improvement of blood glucose control or body weight after treatment with SGLT2 inhibitors. ${ }^{29} 30$ However, studies such as the EMPA-REG OUTCOME trial reported that SGLT2 inhibitors could reduce the ALT values even in patients with suboptimal glucose-lowering or weight-lowering effects, implying that ALT reduction mechanisms might be independent of weight changes or changes in hemoglobin A1c levels. ${ }^{31}{ }^{32}$ Other studies have suggested that potential beneficial hepatic effects of the SGLT2 inhibitors may be attributable to suppression of hepatic inflammation, attenuation of oxidative stress and increase in fatty acid oxidation. ${ }^{33}{ }^{34}$ However, the hypothetical mechanisms have not been well verified.

Third, from the systematic reviews, we found no evidence regarding the association between SGLT2 inhibitor use and reduced risk of solid outcomes such as liver cirrhosis or liver cancer, ${ }^{35}$ mainly because the included clinical trials did not cover a sufficient time period to observe chronic and progressive hepatic outcomes. However, an animal study found SGLT2 inhibitors could induce cell cycle arrest and reduce tumor growth by direct inhibitions of the SGLT2 in tumor cells, and therefore prevent the progression from NASH to hepatocellular carcinoma. ${ }^{36}$ This animal study provides foundational background for a real-world study with a longer observation time to determine the solid outcomes in patients receiving SGLT2 inhibitors.

To the best of our knowledge, this umbrella review of multiple systematic reviews is the first to undertake an evaluation of the methodological quality of included studies focusing on SGLT2 inhibitors for patients with T2D with NAFLD. We found the quality of most included systematic reviews to be low or critically low. Furthermore, the comparators varied among clinical trials in the included systematic reviews, so we suggest the findings should be interpreted with caution. Current evidence supports the idea that SGLT2 inhibitor use is associated with liver enzyme and liver fat reduction in patients with T2D with NAFLD. However, liver improvement outcomes based on liver biopsy from clinical trials of SGLT2 inhibitors remain scarce. Taken together, the effects on weight loss and the associated reduction in risk of cardiovascular and renal events, as demonstrated by studies, ${ }^{3738}$ make the use of SGLT2 inhibitors a potentially rational option for patients with T2D with NAFLD. However, future research is warranted to verify the risks and benefits of SGLT2 inhibitors and to compare longer-term hepatic outcomes with other antidiabetes medications in patients with T2D with NAFLD.

\section{CONCLUSION}

This umbrella overview suggests SGLT2 inhibitors may bring about a reduction in liver enzymes and liver fat, and probably improving liver histology in patients with T2D with NAFLD. However, further studies conforming to high scientific standards are required to bridge the gap in evidence regarding SGLT2 inhibitor use and long-term liver outcomes, such as liver cirrhosis and liver cancer.

\section{Author affiliations}

${ }^{1}$ Department of Pharmacy, Keelung Chang Gung Memorial Hospital, Keelung, Taiwan

${ }^{2}$ School of Pharmacy, Institute of Clinical Pharmacy and Pharmaceutical Sciences, College of Medicine, National Cheng Kung University, Tainan, Taiwan

${ }^{3}$ Division of Sports Medicine, Department of Orthopaedic Surgery, Chiayi Chang Gung Memorial Hospital, Chiayi, Taiwan

${ }^{4}$ College of Medicine, Chang Gung University, Taoyuan, Taiwan

${ }^{5}$ Liver Research Unit, Linkou Chang Gung Memorial Hospital, Taoyuan, Taiwan

${ }^{6}$ Division of Hepatology, Department of Gastroenterology and Hepatology, Linkou Chang Gung Memorial Hospital, Taoyuan, Taiwan

${ }^{7}$ Section of Cardiology, Department of Internal Medicine, Keelung Chang Gung Memorial Hospital, Keelung, Taiwan

${ }^{8}$ Department of Pharmacy, National Cheng Kung University Hospital, Tainan, Taiwan

Contributors The proposal for this study was developed by S-CS and EC-CL and reviewed by all authors. The content of this manuscript was agreed upon by all authors. The descriptive analysis was conducted by S-CS and L-TK. The first draft of the manuscript was developed by S-CS and critically reviewed by all authors. All authors approved the final version of the manuscript before its submission. S-CS is the guarantor of this work.

Funding This study received a grant from Chang Gung Medical Foundation (CMRPG3H1552) and the Ministry of Science and Technology of Taiwan (107-2320-B-006-070-MY3), which had no role in study design, analysis, interpretation or reporting of results.

Competing interests None declared.

Patient consent for publication Not required.

Provenance and peer review Not commissioned; externally peer reviewed.

Data availability statement All data relevant to the study are included in the article or uploaded as supplemental information. 
Supplemental material This content has been supplied by the author(s). It has not been vetted by BMJ Publishing Group Limited (BMJ) and may not have been peer-reviewed. Any opinions or recommendations discussed are solely those of the author(s) and are not endorsed by BMJ. BMJ disclaims all liability and responsibility arising from any reliance placed on the content. Where the content includes any translated material, BMJ does not warrant the accuracy and reliability of the translations (including but not limited to local regulations, clinical guidelines, terminology, drug names and drug dosages), and is not responsible for any error and/or omissions arising from translation and adaptation or otherwise.

Open access This is an open access article distributed in accordance with the Creative Commons Attribution Non Commercial (CC BY-NC 4.0) license, which permits others to distribute, remix, adapt, build upon this work non-commercially, and license their derivative works on different terms, provided the original work is properly cited, appropriate credit is given, any changes made indicated, and the use is non-commercial. See: http://creativecommons.org/licenses/by-nc/4.0/.

ORCID iD

Edward Chia-Cheng Lai http://orcid.org/0000-0002-5852-7652

\section{REFERENCES}

1 Hazlehurst JM, Woods C, Marjot T, et al. Non-Alcoholic fatty liver disease and diabetes. Metabolism 2016;65:1096-108.

2 Younossi ZM, Golabi P, de Avila L, et al. The global epidemiology of NAFLD and NASH in patients with type 2 diabetes: a systematic review and meta-analysis. J Hepatol 2019;71:793-801.

3 Portillo-Sanchez P, Bril F, Maximos M, et al. High prevalence of nonalcoholic fatty liver disease in patients with type 2 diabetes mellitus and normal plasma aminotransferase levels. J Clin Endocrinol Metab 2015;100:2231-8.

4 Loomba R, Wong R, Fraysse J, et al. Nonalcoholic fatty liver disease progression rates to cirrhosis and progression of cirrhosis to decompensation and mortality: a real world analysis of Medicare data. Aliment Pharmacol Ther 2020;51:1149-59.

5 Fiorentino TV, Succurro E, Sciacqua A, et al. Non-Alcoholic fatty liver disease is associated with cardiovascular disease in subjects with different glucose tolerance. Diabetes Metab Res Rev 2020;36:e3333.

6 Wang C, Wang X, Gong G, et al. Increased risk of hepatocellular carcinoma in patients with diabetes mellitus: a systematic review and meta-analysis of cohort studies. Int J Cancer 2012;130:1639-48.

7 Chalasani N, Younossi Z, Lavine JE, et al. The diagnosis and management of non-alcoholic fatty liver disease: practice guideline by the American association for the study of liver diseases, American College of gastroenterology, and the American gastroenterological association. Hepatology 2012;55:2005-23.

8 Cusi K, Orsak B, Bril F, et al. Long-Term pioglitazone treatment for patients with nonalcoholic steatohepatitis and prediabetes or type 2 diabetes mellitus: a randomized trial. Ann Intern Med 2016;165:305-15

9 Schernthaner G, Currie CJ, Schernthaner G-H. Do we still need pioglitazone for the treatment of type 2 diabetes? A risk-benefit critique in 2013. Diabetes Care 2013;36 Suppl 2:S155-61.

10 Snyder HS, Sakaan SA, March KL, et al. Non-Alcoholic fatty liver disease: a review of anti-diabetic pharmacologic therapies. J Clin Transl Hepatol 2018;6:1-7.

11 Chalasani N, Younossi Z, Lavine JE, et al. The diagnosis and management of nonalcoholic fatty liver disease: practice guidance from the American association for the study of liver diseases. Hepatology 2018;67:328-57.

12 Leoni S, Tovoli F, Napoli L, et al. Current guidelines for the management of non-alcoholic fatty liver disease: a systematic review with comparative analysis. World J Gastroenterol 2018;24:3361-73.

13 Romero-Gómez M, Zelber-Sagi S, Trenell M. Treatment of NAFLD with diet, physical activity and exercise. J Hepatol 2017;67:829-46.

14 Targher G, Byrne CD, Tilg $\mathrm{H}$. Nafld and increased risk of cardiovascular disease: clinical associations, pathophysiological mechanisms and pharmacological implications. Gut 2020;69:1691-705.

15 Shao S-C, Chang K-C, Lin S-J, et al. Favorable pleiotropic effects of sodium glucose cotransporter 2 inhibitors: head-to-head comparisons with dipeptidyl peptidase- 4 inhibitors in type 2 diabetes patients. Cardiovasc Diabetol 2020;19:17.

16 Shao S-C, Chang K-C, Chien R-N, et al. Effects of sodium-glucose co-transporter-2 inhibitors on serum alanine aminotransferase levels in people with type 2 diabetes: a multi-institutional cohort study. Diabetes Obes Metab 2020;22:128-34.
17 Shea BJ, Grimshaw JM, Wells GA, et al. Development of AMSTAR: a measurement tool to assess the methodological quality of systematic reviews. BMC Med Res Methodol 2007;7:10.

18 Aromataris E, Fernandez R, Godfrey CM, et al. Summarizing systematic reviews: methodological development, conduct and reporting of an umbrella review approach. Int J Evid Based Healthc 2015;13:132-40.

19 Moher D, Liberati A, Tetzlaff J, et al. Preferred reporting items for systematic reviews and meta-analyses: the PRISMA statement. PLoS Med 2009;6:e1000097.

20 Shea BJ, Reeves BC, Wells G, et al. AMSTAR 2: a critical appraisal tool for systematic reviews that include randomised or nonrandomised studies of healthcare interventions, or both. BMJ 2017;358:j4008.

21 Kumar J, Memon RS, Shahid I, et al. Antidiabetic drugs and nonalcoholic fatty liver disease: a systematic review, meta-analysis and evidence MAP. Dig Liver Dis 2020;8658:30441-2.

22 Tang W, Xu Q, Hong T, et al. Comparative efficacy of anti-diabetic agents on nonalcoholic fatty liver disease in patients with type 2 diabetes mellitus: a systematic review and meta-analysis of randomized and non-randomized studies. Diabetes Metab Res Rev 2016;32:200-16.

23 Xing B, Zhao Y, Dong B, et al. Effects of sodium-glucose cotransporter 2 inhibitors on non-alcoholic fatty liver disease in patients with type 2 diabetes: A meta-analysis of randomized controlled trials. J Diabetes Investig 2020;11:1238-47.

24 Pan CS, Stanley TL. Effect of weight loss medications on hepatic steatosis and steatohepatitis: a systematic review. Front Endocrinol 2020;11:70.

25 Dougherty JA, Guirguis E, Thornby K-A. A systematic review of newer antidiabetic agents in the treatment of nonalcoholic fatty liver disease. Ann Pharmacother 2020;1060028020935105:10600280209 3510.

26 Akuta N, Kawamura Y, Watanabe C, et al. Impact of sodium glucose cotransporter 2 inhibitor on histological features and glucose metabolism of non-alcoholic fatty liver disease complicated by diabetes mellitus. Hepatol Res 2019;49:531-9.

27 Shao S-C, Lin Y-H, Chang K-C, et al. Sodium glucose co-transporter 2 inhibitors and cardiovascular event protections: how applicable are clinical trials and observational studies to real-world patients? BMJ Open Diabetes Res Care 2019;7:e000742.

28 Castellana M, Procino F, Sardone R, et al. Generalizability of sodiumglucose co-transporter-2 inhibitors cardiovascular outcome trials to the type 2 diabetes population: a systematic review and metaanalysis. Cardiovasc Diabetol 2020;19:87.

29 Leiter LA, Forst T, Polidori D, et al. Effect of canagliflozin on liver function tests in patients with type 2 diabetes. Diabetes Metab 2016;42:25-32.

30 Lee PCH, Gu Y, Yeung MY, et al. Dapagliflozin and Empagliflozin ameliorate hepatic dysfunction among Chinese subjects with diabetes in part through glycemic improvement: a single-center, retrospective, observational study. Diabetes Ther 2018;9:285-95.

31 Sattar N, Fitchett D, Hantel S, et al. Empagliflozin is associated with improvements in liver enzymes potentially consistent with reductions in liver fat: results from randomised trials including the EMPA-REG OUTCOME® trial. Diabetologia 2018;61:2155-63.

32 Kuchay MS, Krishan S, Mishra SK, et al. Effect of Empagliflozin on liver fat in patients with type 2 diabetes and nonalcoholic fatty liver disease: a randomized controlled trial (E-LIFT trial). Diabetes Care 2018:41:1801-8.

33 Bonnet F, Scheen AJ. Effects of SGLT2 inhibitors on systemic and tissue low-grade inflammation: the potential contribution to diabetes complications and cardiovascular disease. Diabetes Metab 2018;44:457-64.

34 Mudaliar S, Polidori D, Zambrowicz B, et al. Sodium-Glucose cotransporter inhibitors: effects on renal and intestinal glucose transport: from bench to bedside. Diabetes Care 2015;38:2344-53

35 Scheen AJ. Beneficial effects of SGLT2 inhibitors on fatty liver in type 2 diabetes: a common comorbidity associated with severe complications. Diabetes Metab 2019;45:213-23.

36 Jojima T, Wakamatsu S, Kase M, et al. The SGLT2 inhibitor canagliflozin prevents carcinogenesis in a mouse model of diabetes and non-alcoholic Steatohepatitis-Related hepatocarcinogenesis: association with SGLT2 expression in hepatocellular carcinoma. Int $J$ Mol Sci 2019;20:5237.

37 Stefan N, Häring H-U, Cusi K. Non-Alcoholic fatty liver disease: causes, diagnosis, cardiometabolic consequences, and treatment strategies. Lancet Diabetes Endocrinol 2019;7:313-24.

38 Katsiki N, Perakakis N, Mantzoros C. Effects of sodium-glucose cotransporter-2 (SGLT2) inhibitors on non-alcoholic fatty liver disease/ 
non-alcoholic steatohepatitis: ex quo et quo vadimus? Metabolism 2019;98:iii-ix.

39 Mantovani A, Byrne CD, Scorletti E, et al. Efficacy and safety of anti-hyperglycaemic drugs in patients with non-alcoholic fatty liver disease with or without diabetes: an updated systematic review of randomized controlled trials. Diabetes Metab 2020;3636:30002-1.

40 Raj H, Durgia H, Palui R, et al. SGLT-2 inhibitors in non-alcoholic fatty liver disease patients with type 2 diabetes mellitus: A systematic review. World J Diabetes 2019;10:114-32. 